\title{
FUNCTIONALIZATION OF CELLULOSE AND CHITOSAN IN IONIC LIQUIDS
}

\author{
CRISTINA STEFANESCU, WILLIAM H. DALY and IOAN I. NEGULESCU \\ Department of Chemistry, Louisiana State University, Baton Rouge, LA 70803, USA \\ $\checkmark$ Corresponding author, Grace Drews Lehmann Distinguished Professor Ioan I. Negulescu, \\ inegule@lsu.edu
}

Dedicated with high consideration to Professor Cristofor I. Simionescu on his $100^{\text {th }}$ birth anniversary

\begin{abstract}
Chemistry of cellulose in ionic liquids has been briefly reviewed and, accordingly, the phthalation of chitosan in these ionic solvents has been investigated. Chitosan (K) has been reacted at $100{ }^{\circ} \mathrm{C}$ for 4 hours with phthalic anhydride (PA) in ionic liquids 1-butyl-3-methylimidazolium acetate (BMIMAc) and 1-butyl-3-methylimidazolium chloride (BMIMCl) in the presence of bases, pyridine and 1,4-diazobicyclo[2.2.2] octane (DABCO), or the phthalation has been catalyzed by N-bromosuccinimide (NBS). Depending on the nature of the reaction components, the samples were prepared with molar ratios of PA to anhydroglucose unit (PA:K) from 3:1 to 10:1, including molar ratios of bases or catalyst to chitosan, ranging also from 3:1 to 10:1. All the reaction products were soluble in dimethyl sulfoxide and dimethylformamide. Both functional groups of chitosan units, $-\mathrm{OH}$ and $-\mathrm{NH}_{2}$, reacted, resulting in FTIR confirmed products containing esters, amide, and imide functional groups. Heating the isolated phthalated chitosan products to $200{ }^{\circ} \mathrm{C}$ led to cyclization with the formation of imide groups and elimination of water. When bases controlled the reactions, the highest degrees of substitution of DABCO product $(\mathrm{DS}=0.80)$ was slightly higher than the highest $\mathrm{DC}$ of the reaction products obtained in the presence of pyridine $(\mathrm{DS}=0.77)$. However, the presence of the $\mathrm{N}$ bromosuccinimide catalyst in the system led to an increase of the degree of substitution of the functional groups of chitosan (DS $=1.75$ ), compared with that listed above for the products resulted when the reactions were carried out in the presence of bases. The thermal stability of the chitosan derivatives obtained in the presence of a base depended primarily upon the nature of the counter ion of the ionic liquid. When the reaction was conducted in the acetate ionic liquid BMIMAc, the phthalated chitosan exhibited a lower thermal stability than that of chitosan, while when the chloride ionic liquid BMIMCl was used as solvent, the thermal stability of the phthalated chitosan increased, indicating an interference of the ionic solvents in the mechanisms of reactions. Nevertheless, the thermal behavior of the phthalated products obtained in reactions catalyzed by NBS may be correlated with the increasing degrees of substitution achieved with increased catalyst concentrations: a higher DS resulted in a higher weight loss at higher temperatures.
\end{abstract}

Keywords: 1-butyl-3-methylimidazolium acetate, 1-butyl-3-methylimidazolium chloride, cellulose, chitosan, 1,4diazobicyclo[2.2.2] octane, FTIR, ionic liquids, N-bromosuccinimide, phthalation, phthalic anhydride, pyridine, thermogravimetric analysis

\section{INTRODUCTION}

Working with chitosan, a natural biopolymer, is of interest because it is highly suitable for biomedical and biochemical applications due to its high biocompatibility, biodegradability, and nontoxicity. Chitosan is a biopolymer derived from chitin, a long-chain polymer of $\beta-(1-4)$ linked units of the $\mathrm{N}$-acetyl-glucosamine, which is the second most abundant polysaccharide found in nature after cellulose.

The structure of chitosan is similar to that of cellulose, with two hydroxyl groups (carbon 3 and
6) and an amino group at carbon 2, while cellulose has three hydroxyl groups in its repeating unit. However, since the chitosan is derived from chitin, the N-acetyl-D-glucosamine units formed through the alkaline $N$-deacetylation of the $N$-acetamido functional chitin groups have a typical degree of $70-95 \%$, the rest $(5-30 \%)$ being $\mathrm{N}$-acetyl-glucosamine randomly distributed throughout the polymer chain.

Similarly to cellulose, chitosan polymers have very strong intra and intermolecular hydrogen 
bonding in their structure (Schemes 1 and 2). Therefore, the solubility of cellulose and chitosan is precluded in most of organic solvents.

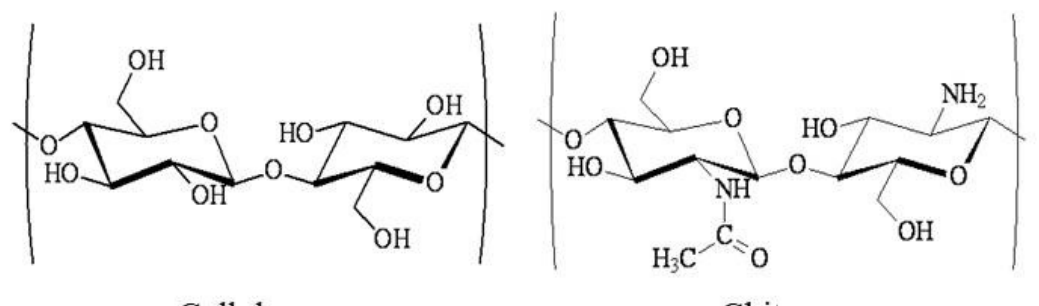

Cellulose

Chitosan

Scheme 1: Structures of cellulose and chitin-derived chitosan

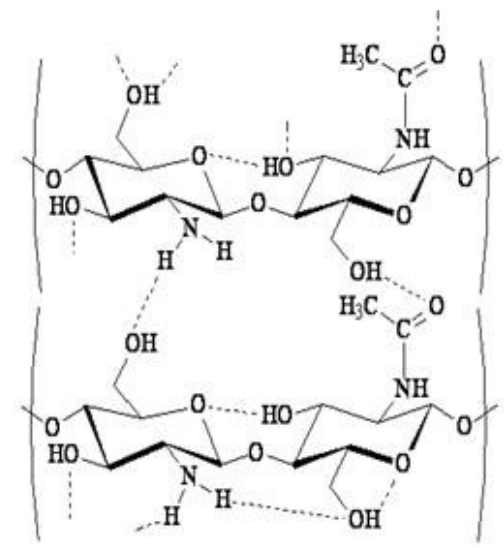

Scheme 2: Inter and intramolecular hydrogen bonding of chitosan polymeric chains

Nonetheless, the chitosan polysaccharide dissolves in nearly all solutions of organic acids at $\mathrm{pH}$ values below 6 . Perhaps the most frequently used organic acids to prepare chitosan solutions are acetic acid and formic acid. In addition, several dilute inorganic acid solutions (e.g. $\mathrm{HNO}_{3}$, $\mathrm{H}_{3} \mathrm{PO}_{4}, \mathrm{HCl}, \mathrm{HClO}_{4}$ ) can be employed to dissolve chitosan.

Cellulose is not soluble in these acidic solvents and therefore a common dissolving organic derivative has been sought, particularly for preparation of chitosan-cellulose composites. ${ }^{1}$ Rather recently, however, a relatively new class of organic solvents, ionic liquids, has been found particularly useful in dissolution of polar organic materials, even polymers, which are otherwise difficult to dissolve. ${ }^{2}$ These solvents are organic salts with the melting point under $100{ }^{\circ} \mathrm{C}$, being able to dissolve rigid chain cellulose under suitable conditions by disrupting the hydrogen bonds. $^{3-6}$ Since cellulose dissolved in pure ionic liquids to an extent of up to $10 \%,{ }^{7}$ ionic liquids have been also similarly considered for chitosan. ${ }^{1}$ The removal of one to two hydrogens from the amino group of the chitosan structure and the replacement with hydrophobic groups results in the destruction of chitosan inherent crystalline structure and the improvement of solubility in general organic solvents. By modifying the chitosan with different reagents, new desired properties can be induced, which will enlarge the field of the potential applications.

The present research focused on the dissolution and functionalization of chitosan in homogeneous ionic liquid solutions. The ionic liquids 1-butyl-3-methylimidazolium acetate (BMIMAc), and 1-butyl-3-methylimidazolium chloride (BMIMCl) were the ionic solvents used to accomplish these goals.

\section{EXPERIMENTAL}

\section{Materials}

All chemicals were used as received, without any further purification. The ionic liquid solvent, 1-butyl3-methylimidazolium acetate (BMIMAc), the catalyst, $\mathrm{N}$-bromosuccinimide (NBS), 1,4-diazobicyclo[2.2.2] octane (DABCO), pyridine, and chitosan $(\mathrm{K})$ with a Brookfield viscosity of $200 \mathrm{~K}$ cps, were purchased from Sigma Aldrich Chemical Company.

The chitosan was dried overnight at $90{ }^{\circ} \mathrm{C}$ and used without any further purification. Dimethyl sulfoxide, DMSO, was obtained from Fisher Scientific. Phthalic anhydride, PA, was acquired from Mallinckrodt. 


\section{Procedures}

Phthalation of chitosan in the presence of $t$-amines

The dissolution of the required amount of $\mathrm{K}$ in ionic liquids in order to form a $2.2 \%$ solution was done by adding the dry chitosan to the respective ionic liquid (BMIMAc or BMIMCl) in a round bottom flask under argon at room temperature and heating it to 100 ${ }^{\circ} \mathrm{C}$. The complete dissolution of $\mathrm{K}$, resulting in an amber solution, lasted 6.5 hours. The reaction of chitosan with PA was performed by adding PA and the base (Pyridine or DABCO) at room temperature to the chitosan solution $(2.2 \%)$ in BMIMAc or BMIMCl required by a molar ratio of $\mathrm{PA}: \mathrm{K}=5: 1$, when the $\mathrm{PA}$ weight was $0.174 \mathrm{~g}$. When performing the reaction in
BMIMAc, the bases used were pyridine for one experiment and $\mathrm{DABCO}$ for a separate one, with the molar ratio of the base to $K$ of $3: 1,5: 1$, or $10: 1$. In the case of BMIMC, only one base was used, pyridine, with a molar ratio of either $2: 1$ or of $5: 1$. In each experiment, the reaction was allowed to proceed at 100 ${ }^{\circ} \mathrm{C}$ for 4 hours. After cooling the solution to room temperature, the polymer was precipitated in methanol (200 mL), filtered and then washed thoroughly on the filter with methanol (400 mL). The phthalated chitosan collected was dried first at $45{ }^{\circ} \mathrm{C}$ for 24 hours and then under vacuum at $50{ }^{\circ} \mathrm{C}$ for 2 hours for a complete removal of methanol.

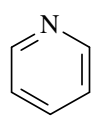

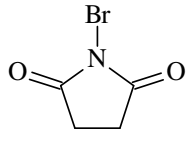

b

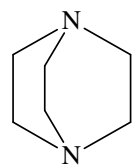

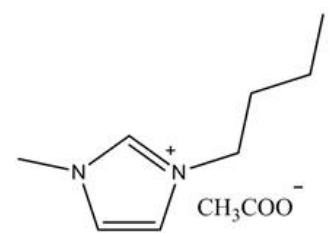

Scheme 4: Chemical structure of 1-butyl-3methylimidazolium acetate (BMIMAc) and 1-butyl-3methylimidazolium chloride (BMIMCl) ionic liquids

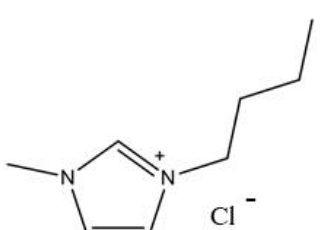
bromosuccinimide (NBS) (b), and 1,4 diazobicyclo[2.2.2] octane (DABCO) (c)

1-Butyl-3-methylimidazolium chloride (BMIMCl) was synthesized from 1-methyl imidazol and chlorobutane. ${ }^{8}$

\section{Phthalation of chitosan in the presence of NBS catalyst}

The reaction of chitosan with PA in BMIMAc in the presence of the catalyst proceeded homogeneously. It was performed as follows: PA $(0.165 \mathrm{~g})$ and NBS $(0.198 \mathrm{~g})$ were dissolved in DMSO $(2 \mathrm{~mL})$ in order to reduce the viscosity at room temperature and the solution was added to the required amount of ionic solution of chitosan $(2.2 \%)$ according to the molar ratios of PA:K $(3: 1,5: 1$, or $7: 1)$ and of NBS:K (3:1, $7: 1$, and 10:1). The reaction was allowed to proceed at $100{ }^{\circ} \mathrm{C}$ for 4 hours under magnetic stirring. After cooling the solution to room temperature, the reaction product was separated and processed as described above.

\section{Analyses}

The resulted phthalated chitosan outcomes were characterized spectroscopically by FTIR (ThermoNicolet 300) using $\mathrm{KBr}$ pellets. Thermogravimetric analysis (TGA) was performed on a TA Q5000 (TA Instruments), with a heating rate of $10{ }^{\circ} \mathrm{C} / \mathrm{min}$.

\section{RESULTS AND DISCUSSION}

\section{Chemistry of cellulose in ionic liquid solutions}

As mentioned before, due to the presence of three hydroxyl groups in its structure, cellulose has intermolecular and intramolecular hydrogen bonding, which makes its dissolution impossible in most organic solvents. On the other hand, cellulose is soluble in very polar organic $\mathrm{N}$ oxides, such as the monohydrate of N-methyl Morpholine N-oxide, ${ }^{9}$ and in completely dissociated ionic liquids, which have been reported as media for its functionalization, such as acetylation, ${ }^{10,11}$ esterification, ${ }^{12,13}$ etherification ${ }^{14}$ and carboxymethylation. ${ }^{15}$

For example, acetylation of cellulose in 1butyl-3-methylimidazolium chloride using acetic acid anhydride in the presence of pyridine yielded soluble products of controlled degree of substitution (Scheme 5). ${ }^{16}$

Nonetheless, the chemical structure of the ionic liquid might interfere with the reactants, giving rise to different reaction compounds. When trityl cellulose was synthesized by performing the reaction of trityl chloride in excess with cellulose in 1-butyl-3-methylimidazolium chloride as solvent, the addition of a base was necessary in order to prevent the degradation of cellulose by capturing the hydrogen chloride formed during the reaction and a degree of substitution of 1.0 has been obtained. ${ }^{14}$ Surprisingly, when the same treatment of cellulose with trityl chloride was performed in a different ionic liquid, 1-ethyl-3methylimidazolium acetate, cellulose acetate was obtained instead of trityl cellulose. This behavior 
was explained by the formation of reactive trityl acetic acid esters as intermediate, which will undergo a transesterification reaction with cellulose to give cellulose acetate as final product. $^{17}$

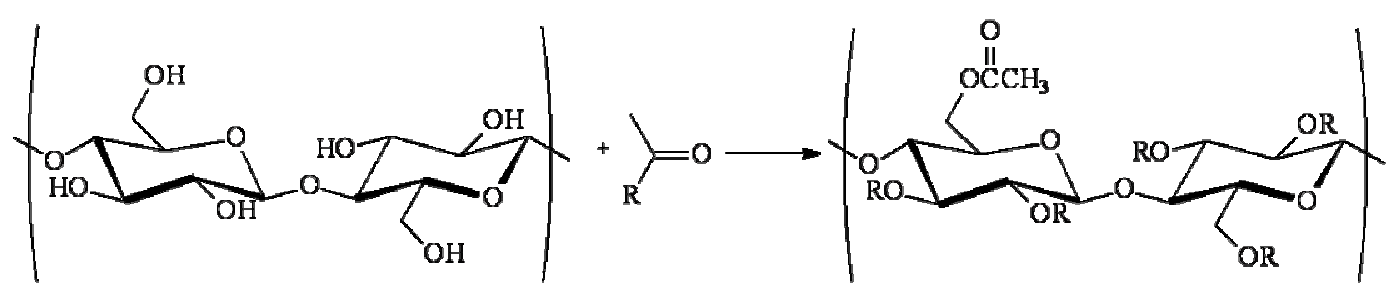

Scheme 5: Acetylation of cellulose with acetic anhydride: $\mathrm{R}$ is $\mathrm{H}-$ or $\mathrm{CH} 3 \mathrm{CO}-$

Similarly, the homogeneous chemical modification of cellulose with phthalic anhydride in the presence of 1-allyl-3-methylimidazolium chloride ionic liquid and in the absence of a catalyst resulted in phthalated cellulosic derivatives with a degree of substitution ranging from 0.10 to 0.73 , depending on the reaction conditions, while when the same reaction was performed in 1-butyl-3-methylimidazolium chloride (BMIMAc), higher degrees of substitution, ranging from 0.12 to 2.54 , were reported. ${ }^{12}$

\section{Chemistry of chitosan in ionic liquid solutions}

After reviewing the reactions of cellulose in ionic liquids, we have extended the work on the chemistry of chitosan in ionic liquids and observed significant differences depending particularly on the catalytic settings of the reactions.

\section{Nucleophilic reactivity of chitosan in ionic liquids promoted by tert-amines}

Experimentally, chitosan $(\mathrm{K})$ has been reacted with PA in the presence of a base (pyridine or $\mathrm{DABCO}$ ) using either 1-butyl-3methylimidazoilium chloride (BMIMCl) or 1butyl-3-methylimidazolium acetate (BMIMAc) as ionic solvent. The reactions in either BMIMCl or BMIMAc proceeded homogeneously. Both functional groups of chitosan, $-\mathrm{OH}$ and $-\mathrm{NH}_{2}$ reacted, resulting in products containing esters, amide, and imide functional groups as presented in Scheme 6. The mechanism of the reaction is displayed in Scheme 7. ${ }^{1}$

The reaction products were characterized spectroscopically by FTIR and by the thermographic analysis.

\section{FTIR analysis}

The new peaks seen in the FTIR spectra of the phthalated chitosan (when BMIMAc was used as ionic solvent), as compared to the chitosan peaks, indicate that the reaction proceeded as described in Figure 1. The absorption band at $723 \mathrm{~cm}^{-1}$ is a characteristic of ortho-disubstituted aromatic rings, suggesting that indeed the PA reacted with the chitosan.

The intense peaks at $1716 \mathrm{~cm}^{-1}$ and $1654 \mathrm{~cm}^{-1}$ are attributed to the carbonyl stretch in esters and carboxylic acids. Both peaks have been shifted to a lower frequency due to the conjugation of the carbonyl group with the aromatic ring attached to it and due to the presence of intermolecular hydrogen bonding. The absorption band at 1654 $\mathrm{cm}^{-1}$ is also attributed to the carbonyl stretch in amides. The N-H bending for amide can be identified at $1561 \mathrm{~cm}^{-1}$.

A carbonyl stretch for imides can be seen at $1775 \mathrm{~cm}^{-1}$. The imide group is a result of the cyclization shown in Scheme 6. The presence of all these peaks confirms that both $-\mathrm{OH}$ and $-\mathrm{NH}_{2}$ of the chitosan anhydroglucose unit reacted with PA.

From these FTIR spectra for the reaction of chitosan with PA in the presence of pyridine or $\mathrm{DABCO}$, it can be observed that, in the presence of $\mathrm{DABCO}$, the absorption intensities of the DABCO film are greater than those of the pyridine film. Both resulting products were soluble in DMSO. When the DABCO films used for the FTIR were heated to $200{ }^{\circ} \mathrm{C}$ for 3 hours and the FTIR spectra were recorded again, an increase in the intensity of the peaks corresponding to imide and ortho-disubstituted aromatic rings and a decrease in the intensity of the peaks for carbonyl stretch and $\mathrm{N}-\mathrm{H}$ bending for amides can be noticed. This occurrence is the 
Polysaccharides

result of cyclization with the formation of imide

groups (Fig. 1 bottom).
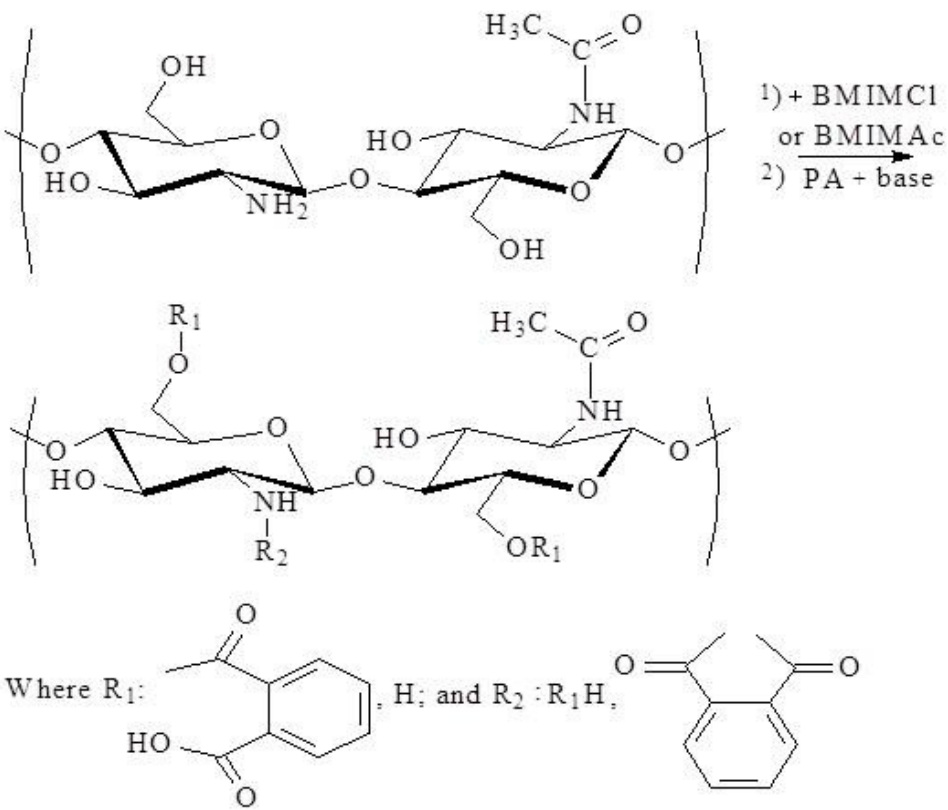

Scheme 6: Reaction of chitosan with phthalic anhydride

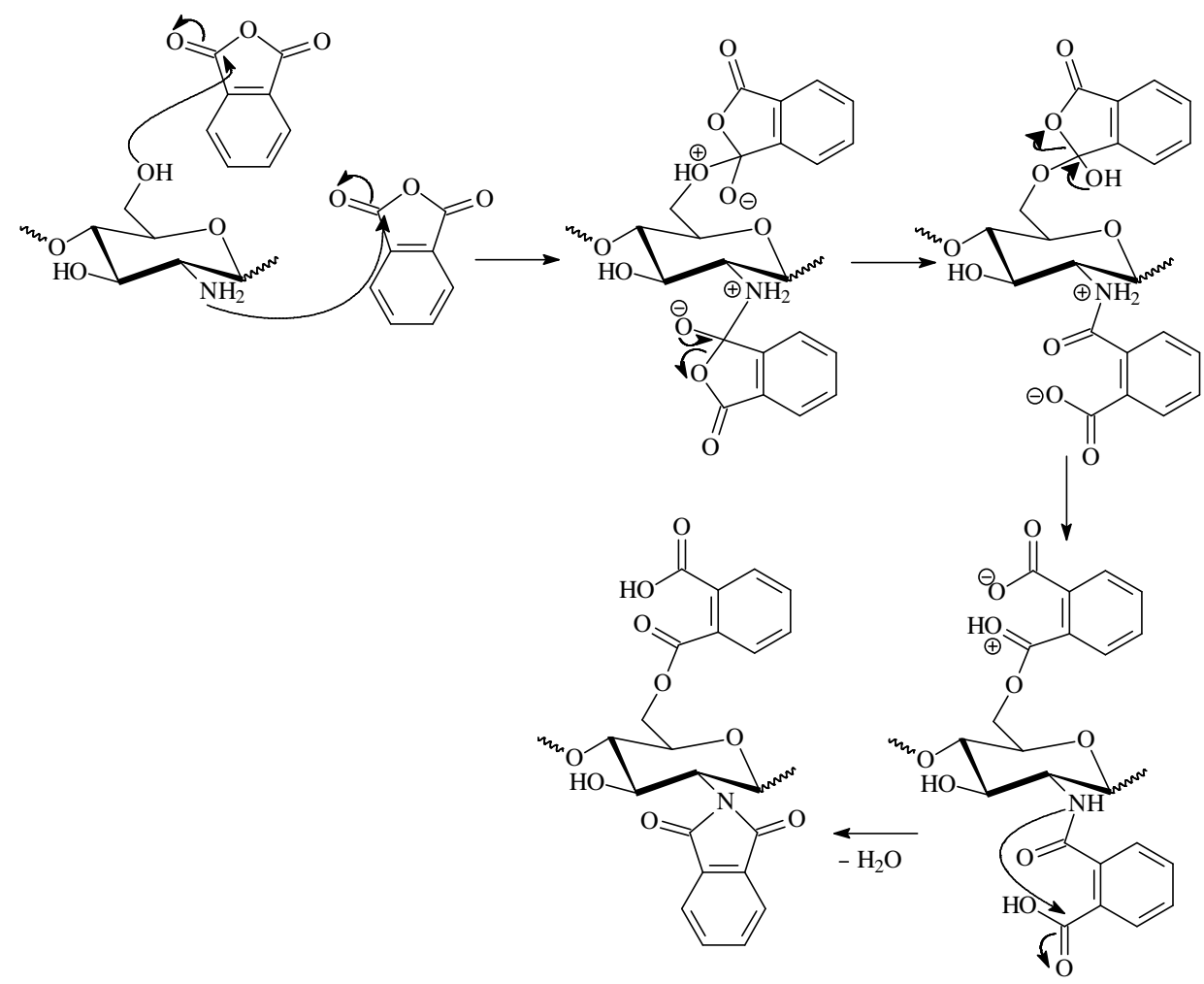

Scheme 7: Mechanistic route towards the synthesis of phthalated chitosan 


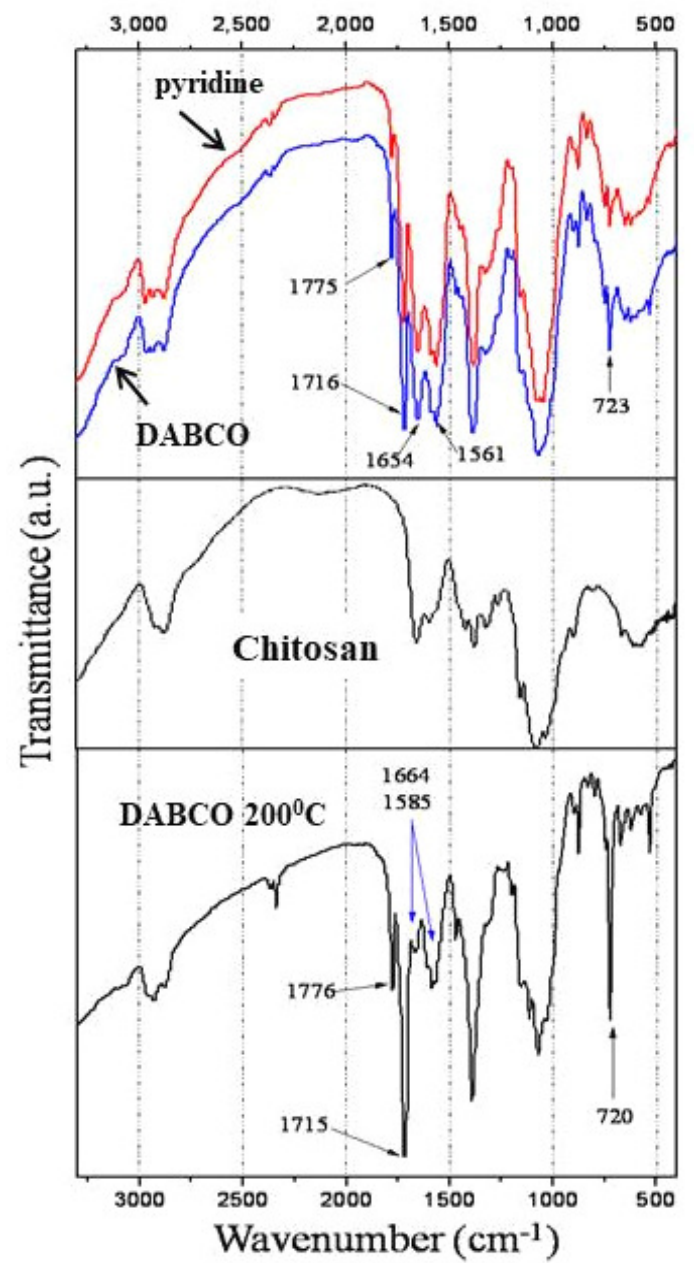

Figure 1: FTIR spectra of chitosan reacted with PA in BMIMAc using pyridine or DABCO (above), of unreacted chitosan (middle) and after heating the $\mathrm{KBr}$ pellets containing the DABCO sample to $200{ }^{\circ} \mathrm{C}$ for 3 hours (bottom)

The degrees of substitution (DS) of the reaction products were calculated based on the calibration curves obtained from the FT-IR spectra of physical mixtures of N-methyl phthalimide with chitosan. ${ }^{18}$ The DABCO outcomes displayed slightly higher degrees of substitution (the largest DS was 0.80 , for the reaction product obtained with a molar ratio of PA to chitosan of 5:1 and of DABCO to chitosan of 10:1) than the DS of the reaction products obtained in the presence of pyridine (the largest DS $=0.77$, of the product obtained with the molar ratio of PA to chitosan of 5:1 and of pyridine to chitosan of 10:1).

\section{Thermal analysis}

The reaction of chitosan with phthalic anhydride reduced the thermal stability of the reaction products (Fig. 2). The onset temperatures of degradation from TG curves were $275{ }^{\circ} \mathrm{C}$ (chitosan, K), $238{ }^{\circ} \mathrm{C}$ (phthalated chitosan $2 \mathrm{~h}-$ KPA 2h) and $226{ }^{\circ} \mathrm{C}$ (phthalated chitosan $4 \mathrm{~h}-$ KPA 4h), with maximum rates (DTG) at $90{ }^{\circ} \mathrm{C}$ and $300{ }^{\circ} \mathrm{C}(\mathrm{K}), 93{ }^{\circ} \mathrm{C}$ and $253{ }^{\circ} \mathrm{C}($ KPA $2 \mathrm{~h}$ ) and $93{ }^{\circ} \mathrm{C}$ and $245{ }^{\circ} \mathrm{C}$ (KPA 4h). The maximum rates at $90{ }^{\circ} \mathrm{C}$ and $93{ }^{\circ} \mathrm{C}$ are assigned to the water loss. The maximum rates at $253{ }^{\circ} \mathrm{C}$ and $245{ }^{\circ} \mathrm{C}$ of the phthalated chitosans are attributed to the degradation of the polymer backbone, as well as the degradation of amide and imide substituted units. At 50\% weight loss, the decomposition temperature of $\mathrm{K}\left(330{ }^{\circ} \mathrm{C}\right)$ is higher than those of phthalated chitosan samples KPA $2 \mathrm{~h}\left(320^{\circ} \mathrm{C}\right)$ and KPA $4 \mathrm{~h}\left(312{ }^{\circ} \mathrm{C}\right)$, respectively. The same trend was observed for the thermal residue values: $31 \%$ for K, $29 \%$ for KPA $2 \mathrm{~h}$ and $27 \%$ for KPA $4 \mathrm{~h}$.

The thermal stability of the chitosan derivatives depended primarily upon the nature of the counter ion of the ionic liquid (Fig. 3). When the reaction was conducted in the acetate ionic 
liquid BMIMAc (Fig. 3 A), the phthalated chitosan exhibited lower thermal stability than chitosan (B). When the chloride ionic liquid $\mathrm{BMIMCl}$ was used as solvent, the thermal stability of the chitosan adducts increased (Fig. 3 C). The onset temperatures of degradation were $302{ }^{\circ} \mathrm{C}$ and $312{ }^{\circ} \mathrm{C}$ for the chitosan reacted with $\mathrm{PA}$ in $\mathrm{BMIMCl}$ chloride ionic liquid using pyridine in a molar ratio of $1: 2$ and $1: 5$, respectively (Fig. $3 \mathrm{C}$ ), higher than that of the

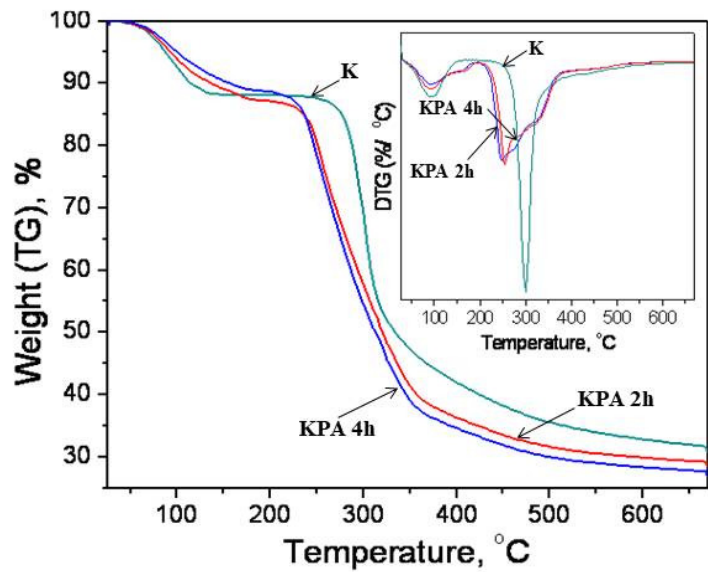

Figure 2: Thermogravimetric traces (TG) and corresponding derivatives for chitosan $(\mathrm{K})$, phthalated chitosan $2 \mathrm{~h}$ (KPA 2h) and phthalated chitosan $4 \mathrm{~h}$ (KPA 4h)

It can be concluded that the reaction of chitosan with phthalic anhydride in the presence of a base and using ionic liquids as solvating media occurred at both $-\mathrm{OH}$ and $-\mathrm{NH}_{2}$ groups. Chemical modification of chitosan using the chloride ionic liquid resulted in products with higher thermal stability than that of the reaction products obtained in the acetate ionic liquid. Heating the isolated phthalated chitosans to 250 ${ }^{\circ} \mathrm{C}$ promoted cyclization, with the formation of imide groups and elimination of water. The FTIR spectroscopic data supported this supposition. chitosan reacted with PA in BMIMAc acetate ionic liquid using DABCO and pyridine, $224{ }^{\circ} \mathrm{C}$ and $228{ }^{\circ} \mathrm{C}$, respectively (Fig. 3A), and also higher than that of unreacted chitosan, $279{ }^{\circ} \mathrm{C}$ (Fig. $3 \mathrm{~B}$ ). The weight loss up to $100{ }^{\circ} \mathrm{C}$ for chitosan (Fig. $3 \mathrm{~B}$ ) is attributed to the loss of water, while the loss to $250{ }^{\circ} \mathrm{C}$ registered for the reaction products (Fig. $3 \mathrm{~A}$ and $3 \mathrm{C}$ ) resulted from the cyclization and formation of imide groups, as confirmed by the FTIR studies.

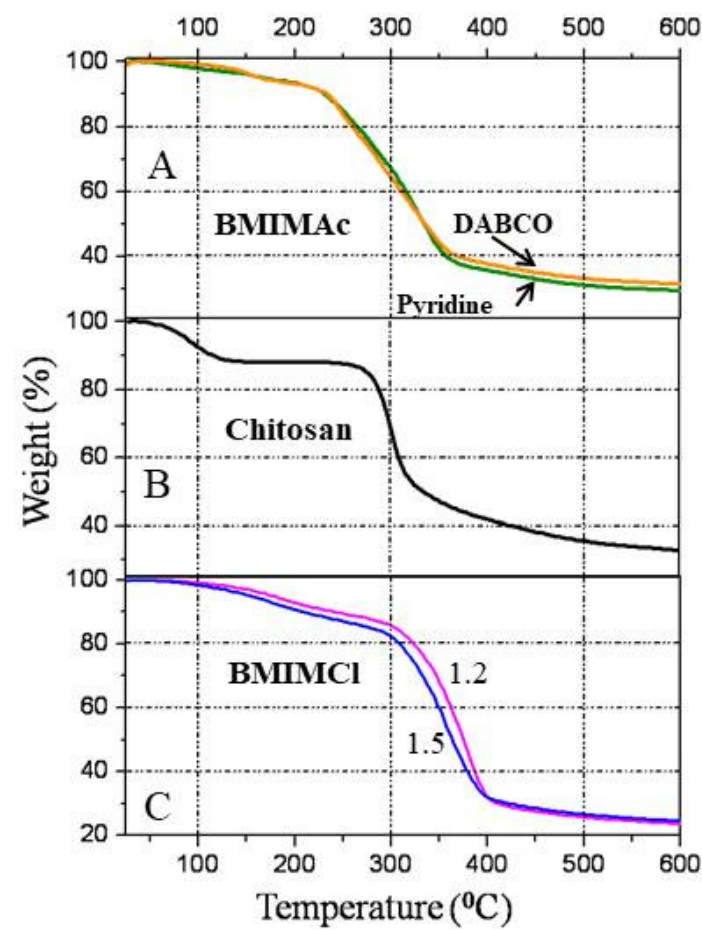

Figure 3: Thermogravimetric traces of chitosan (B) and of products of chitosan reacted with PA (molar ratio of PA to chitosan 5:1) in BMIMAc using pyridine or DABCO as a base; (A) and in BMIMCl with a molar ratio of chitosan to pyridine of $1: 5$ or $1: 2$ (C)

\section{Functionalization of chitosan in ionic liquids promoted by catalysts}

$N$-Bromosuccinimide proved to be a highly effective catalyst for the acylation of alcohols, ${ }^{19}$ and accordingly for homogeneous modification of $-\mathrm{OH}$ groups containing cellulose, ${ }^{20,21}$ and hemicelluloses. ${ }^{22}$

As presented in the following, NBS was also a very efficient catalyst for phthaloylation of chitosan. The reaction of chitosan with PA in BMIMAc catalyzed by NBS proceeded homogeneously. Both $-\mathrm{OH}$ and $-\mathrm{NH}_{2}$ functional 
groups of chitosan reacted, resulting in products containing esters, amide, and imide functional groups (Scheme 8).

The role of NBS is not clear, but a possible explanation is that NBS acts as a source for $\mathrm{Br}^{+}$, which in turn activates the carbonyl groups of PA to produce highly reactive acylating agent, as shown in Scheme 9. ${ }^{1}$ The acylating agent reacts with the hydroxyl and amino groups of chitosan, which upon the elimination of NBS produces phthaloylated chitosan. This hypothesis, however, needs further investigation to determine exactly the actual role of the NBS reagent.

The reaction products were characterized spectroscopically by FTIR and by thermogravimetric analysis (TGA).

\section{FTIR analysis}

The presence of new peaks in the FTIR spectra of the phthalated $\mathrm{K}$ obtained in BMIMAc and using NBS as catalyst presented in Figure 4 attests that the reaction proceeded as described in Scheme 9. Accordingly, when the chitosan $-\mathrm{OH}$ groups reacted with PA, ester groups were formed. During this reaction, carboxylic acids were also developed.

The absorption peaks in the FTIR spectra at $1716 \mathrm{~cm}^{-1}$ and $1655 \mathrm{~cm}^{-1}$ correspond to the carbonyl stretch in the above mentioned esters and carboxylic acid groups. Both peaks have been shifted to a lower frequency due to the conjugation of the carbonyl group with the aromatic ring attached to it and to the presence of intermolecular hydrogen bonding as well. The reaction with $\mathrm{PA}$ of $-\mathrm{NH}_{2}$ groups of chitosan resulted in the development of amide and imide groups. The formation of imide groups is the result of cyclization, as shown in Figure 4 A.

The carbonyl stretch for the imide and amide groups is represented by the bands at $1777 \mathrm{~cm}^{-1}$ and $1655 \mathrm{~cm}^{-1}$, respectively. The N-H bending for amide is identified at $1561 \mathrm{~cm}^{-1}$. The absorption band at $723 \mathrm{~cm}^{-1}$ is a characteristic for orthodisubstituted aromatic rings, suggesting again that the PA reacted with the chitosan (Fig. 4 A). When the pellets used for the FTIR were heated to 200 ${ }^{\circ} \mathrm{C}$ for 2 hours and spectra were recorded again, an increase in the intensity of the peaks corresponding to imide and ester groups and a decrease in the intensity of the peaks for carbonyl stretch and $\mathrm{N}-\mathrm{H}$ bending for amides were observed. This behavior is the result of cyclization with the formation of imide groups (Fig. 4 C).

When the amount of catalyst, NBS, was increased from a molar ratio of NBS to anhydroglucose unit of $\mathrm{K}$ of $3: 1$ to $7: 1$ and 10:1, while keeping constant the molar ratio of PA to $\mathrm{K}$ as PA:K $=3$, an enhancement in the intensity of the peaks in the FTIR spectra corresponding to the reacted $\mathrm{K}$ is observed.

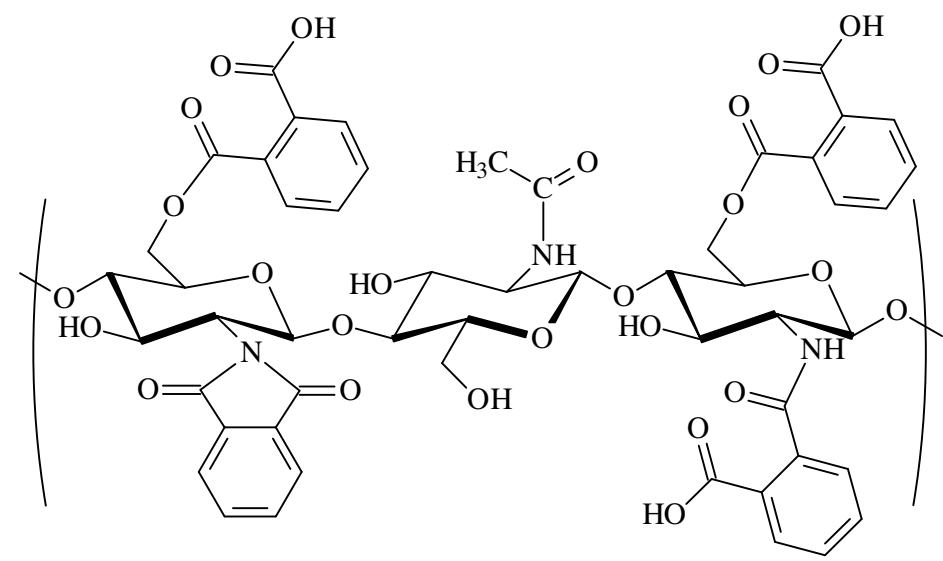

Scheme 8: Structure of chitosan reacted with phthalic anhydride in the presence of NBS

Table 1 lists the ratio of the newly formed peaks in the phthalated $\mathrm{K}$ to the $\mathrm{C}-\mathrm{O}-\mathrm{C}$ bridge symmetric stretching $\left(1076 \mathrm{~cm}^{-1}\right)$. It can be seen that the intensity of the peaks corresponding to the imide and ester groups is increasing with the increase of the catalyst amount added to the reaction system. At the same time, a decrease in the absorption bands of carboxylic acids, amide I and amide II is observed. The same trend could be 
identified when the pellets used for the FTIR spectra were heated to $200{ }^{\circ} \mathrm{C}$ for 2 hours.

The degrees of substitution (DS) of the reaction products heated to $20{ }^{\circ} \mathrm{C}$, calculated as described above based on the calibration curves obtained from the FT-IR spectra, ranged from
0.65 (PA:K = 3:1 and NBS:K = 3:1) to 1.75 (PA:K = 7:1 and NBS:K=10:1). The DS of 1.75 was the highest substitution degree obtained in the reaction of chitosan with phthalic anhydride in ionic liquids.<smiles>CC1CC(=O)N(C(=O)c2ccccc2C(=O)OBr)C1=O</smiles>

Acylating agent
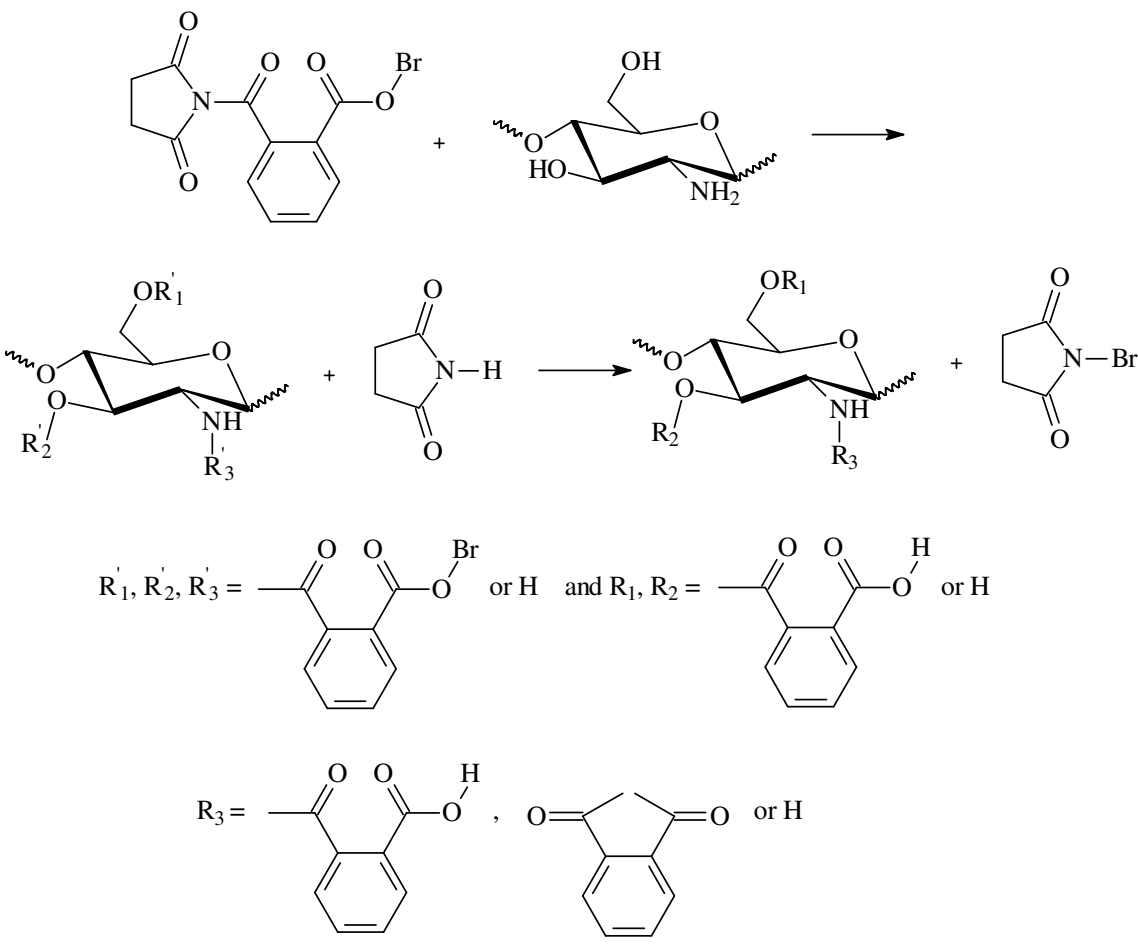

Scheme 9: Mechanism of phthaloylation of chitosan using NBS as catalyst

Table 1

Ratios of newly formed absorption peaks in phthalated chitosan to the C-O-C bridge symmetric stretching peak $\left(1076 \mathrm{~cm}^{-1}\right)^{*}$

\begin{tabular}{ccccc}
\hline $\begin{array}{l}\text { Experimental NBS:K } \\
\left(100^{\circ} \mathrm{C}, 4 \mathrm{~h}\right)\end{array}$ & Imide/CO & Ester/CO & Carboxylic acid/CO & Amide II/CO \\
\hline $3: 1$ & 0.33 & 0.92 & 0.96 & 0.98 \\
$7: 1$ & 0.47 & 1.55 & 0.61 & 0.48 \\
$10: 1$ & 0.63 & 1.59 & 0.48 & 0.29 \\
\hline Experimental NBS:K & Imide/CO & Ester/CO & Carboxylic acid/CO & Amide II/CO \\
\hline (after 2 h at $\left.200{ }^{\circ} \mathrm{C}\right)$ & 0.55 & 1.28 & 0.58 & 0.64 \\
$7: 1$ & 0.58 & 1.76 & 0.39 & 0.30 \\
$10: 1$ & 0.67 & 1.61 & 0.37 & 0.22 \\
\hline
\end{tabular}

* The molar ratio of NBS:K used was 3:1, 7:1, and 10:1, while the molar ratio of PA:K was kept constant to $3: 1$ 


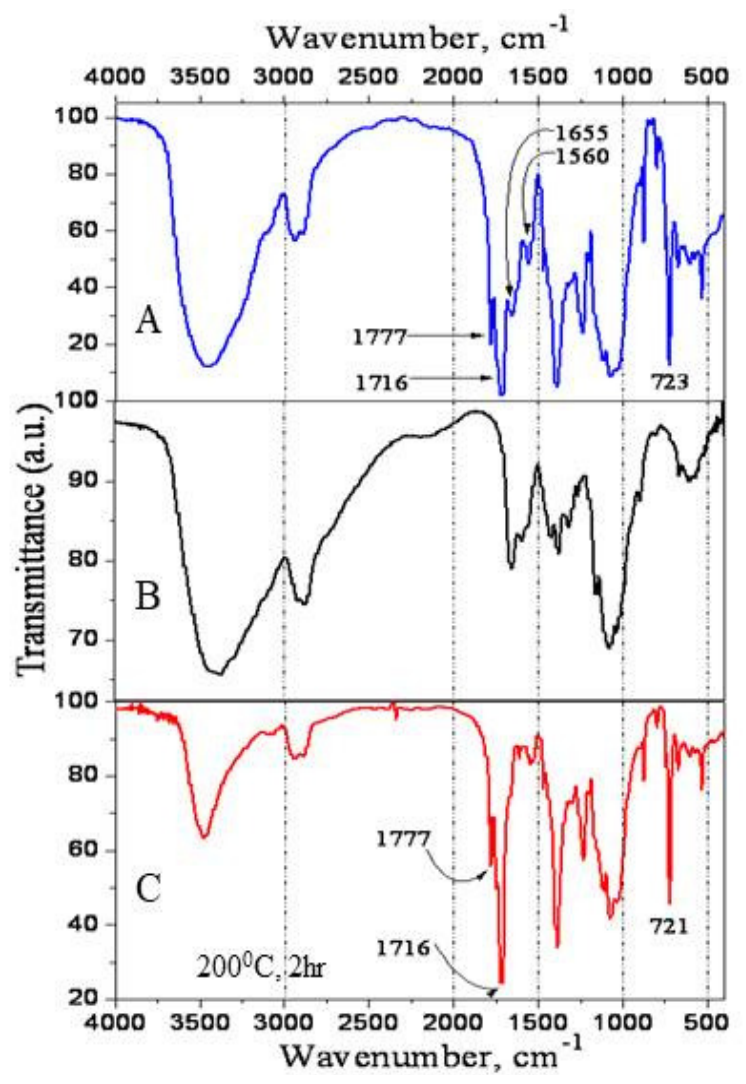

Figure 4: FT-IR spectra of (A) chitosan reacted in BMIMAc with PA in the presence of NBS, at the molar ratio of K to PA and of K to NBS of 3:1 and 10:1, respectively; (B) unreacted chitosan; and (C) red spectrum of phthalated chitosan heated to $200{ }^{\circ} \mathrm{C}$ for 2 hours

\section{Thermal analysis}

The thermal behavior of chitosan and phthalated chitosan obtained in the presence of NBS as catalyst is presented by Figure 5. The thermograms plotted in this figure exhibit an increase/decrease of the onset temperatures of degradation $\left(\mathrm{T}_{\mathrm{d}}\right)$ for the reaction products, when compared to that of unreacted chitosan, depending mostly on the experimental conditions, particularly on the NBS:K ratios.

For example, when the amount of PA was kept constant (PA:K = 3:1) and the molar ratio of NBS:K was changed from 7:1 (thermogram C) to 10:1 (thermogram D), the onset temperatures of degradation $\mathrm{T}_{\mathrm{d}}$ were $288{ }^{\circ} \mathrm{C}$ and, respectively, 310 ${ }^{\circ} \mathrm{C}$, higher than that of chitosan (thermogram A), $\mathrm{T}_{\mathrm{d}}=280{ }^{\circ} \mathrm{C}$. Otherwise, for a lower catalyst concentration, viz. NBS:K = 3:1 (thermogram B), a lower $\mathrm{T}_{\mathrm{d}}$, of $248{ }^{\circ} \mathrm{C}$, was registered. The same trend was observed for the thermal stability of the reaction products up to $350{ }^{\circ} \mathrm{C}$. However, if one compares the residual weights at $500{ }^{\circ} \mathrm{C}$, the unreacted chitosan sample had a higher weight of the residuum (39\% ash) than that of all other reacted products, the ash weight of which dropped with the increased NBS:K ratio from 33\% to $31 \%$ for the NBS:K ratios of 3:1 and 7:1 (thermograms $B$ and C, respectively), to the lowest of $19 \%$ for the NBS:K ratio of 10:1 (thermogram D). This thermal behavior may be correlated with the degrees of substitution of the reacted chitosan products obtained in reactions catalyzed with increased catalyst concentrations. Therefore, a higher DS will result in a higher weight loss at higher temperatures.

As in the preceding case of reactions of chitosan with PA in the presence of tert-amines, the weight loss up to $150{ }^{\circ} \mathrm{C}$ of the catalyzed phthalation products is attributed to the loss of water as a result of cyclization and formation of imide groups, as confirmed by the FTIR studies. 
Polysaccharides

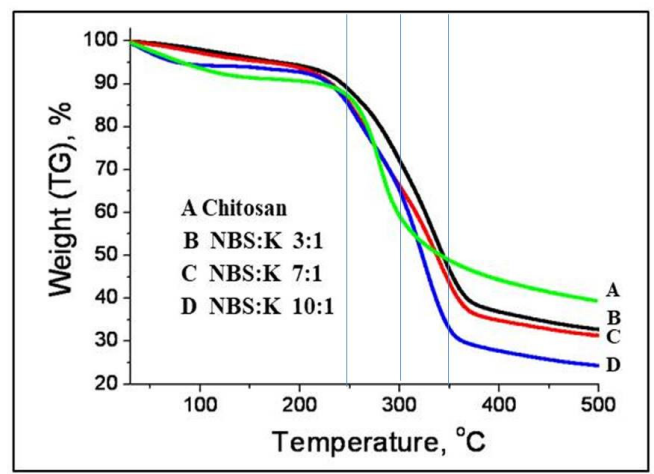

Figure 5: Thermogravimetric traces (TG) for K (A) and K reacted with PA (the molar ratio of NBS:K was 3:1 (B), 7:1 (C), and 10:1 (D), respectively, while the molar ratio of PA:K was constant (3:1))

\section{CONCLUSION}

The reaction of chitosan with PA in either $\mathrm{BMIMCl}$ or BMIMAc in the presence of a base, pyridine or DABCO, or catalyzed by $\mathrm{N}$ bromosuccinimide, proceeded homogeneously. All the reaction products were soluble in dimethyl sulfoxide. Both functional groups of chitosan polymer units, $-\mathrm{OH}$ and $-\mathrm{NH}_{2}$, reacted, resulting in FTIR confirmed products containing esters, amide, and imide functional groups. Heating the isolated phthalated chitosan adducts to $200{ }^{\circ} \mathrm{C}$ promoted the cyclization with the formation of imide groups by elimination of water.

When the phthalation reactions were controlled by bases, the highest degree of substitution of DABCO products was slightly higher than the highest DS of the reaction products obtained in the presence of pyridine. However, the presence of a the $N$ bromosuccinimide catalyst in the system led to an increase in the degree of substitution of the functional groups of chitosan, compared with that of the products of the reactions carried out in the presence of bases.

The thermal stability of the chitosan derivatives obtained in the presence of a base depended primarily upon the nature of the counter ion of the ionic liquid. When the reaction was conducted in the acetate ionic liquid BMIMAc, the phthalated chitosan exhibited a lower thermal stability than that of chitosan, while when the chloride ionic liquid BMIMCl was used as solvent, the thermal stability of the phthalated chitosan outcomes increased, indicating the interference of the ionic solvents in the mechanisms of reactions. Nevertheless, the thermal behavior of the phthalated products obtained in reactions catalyzed by NBS may be correlated with the increasing degrees of substitution achieved with increased catalyst concentrations: a higher DS resulted in a higher weight loss at higher temperatures.

ACKNOWLEDGEMENTS: This paper is dedicated with high consideration to Professor Cristofor I. Simionescu, "the academic father" of one of the co-authors (IN). The USDA MultiState S-1075 Project "The Science and Engineering for a Biobased Industry and Economy is also acknowledged for the efforts in manuscript preparation (IN).

\section{REFERENCES}

1 C. Stefanescu, $\mathrm{PhD}$ Thesis, Louisiana State University, Baton Rouge, LA, 2010, https://digitalcommons.lsu.edu/gradschool_dissertation s/2692/

2 R. D. Rogers and K. R. Seddon, Science, 302, 792 (2003), https://doi.org/10.1126/science.1090313

3 R. P. Swatloski, S. K. Spear, J. D. Holbrey and R. D. Rogers, J. Am. Chem. Soc., 124, 4974 (2002), https://doi.org/10.1021/ja025790m

4 S. D. Zhu, Y. X. Wu, Q. M. Chen, Z. N. Yu, C. W. Wang et al., Green Chem., 8, 325 (2006), http://dx.doi.org/10.1039/b601395c

5 V. M. Egorov, S. V. Smirnova, A. A. Formanovsky, I. V. Pletnev and Y. A. Zolotov, Anal. Bioanal. Chem., 387, $2263 \quad$ (2007), https://doi.org/10.1007/s00216-006-1114-X

6 R. C. Remsing, R. P. Swatloski, R. D. Rogers and G. Moyna, Chem. Commun., 42, 1271 (2006), https://doi.org/10.1039/B600586C

7 K. Schlufter, H. P. Schmauder, S. Dorn and T. Heinze, Macromol. Rapid Commun., 27, 1670 (2006), https://doi.org/10.1002/marc.200600463 


\section{CRISTINA STEFANESCU et al.}

8 P. J. Dyson, M. C. Grossel, N. Srinivasan, T. Vine, T. Welton et al., J. Chem. Soc.-Dalton Trans., 19, 3465 (1997), https://doi.org/10.1039/A702978K

9 S. Petrovan, J. R. Collier and I. I. Negulescu, J. Appl. Polym. Sci., 79, $396 \quad$ (2001), https://doi.org/10.1002/10974628(20010118)79:3<396::AID-APP20>3.0.CO;2-E

10 T. Heinze, K. Schwikal and S. Barthel, Macromol. Biosci., 5, $\quad 520 \quad$ (2005), https://doi.org/10.1002/mabi.200500039

11 J. Wu, J. Zhang, H. Zhang, J. He, Q. Ren et al., Biomacromolecules, $\quad 5, \quad 266 \quad$ (2004), https://doi.org/10.1021/bm034398d

12 C. F. Liu, R. C. Sun, A. P. Zhang, M. H. Qin, J. L. Ren et al., J. Agric. Food Chem., 55, 2399 (2007), https://doi.org/10.1021/jf062876g

13 C. F. Liu, R. C. Sun, A. P. Zhang and J. L. Ren, Carbohyd. Polym., 68, $17 \quad$ (2007), https://doi.org/10.1016/j.carbpol.2006.07.002

14 T. Erdmenger, C. Haensch, R. Hoogenboom and U. S. Schubert, Macromol. Biosci., 7, 440 (2007), https://doi.org/10.1002/mabi.200600253

15 P. White, in "Regenerated Cellulose Fibres", edited by C. Woodings, Woodhead Publishing, 2001, pp. 6287
16 S. Barthel and T. Heinze, Green Chem., 8, 301 (2006), https://doi.org/10.1039/B513157J

17 S. Kohler, T. Liebert, M. Schobitz, J. Schaller, F. Meister et al., Macromol. Rapid Commun., 28, 2311 (2007), https://doi.org/10.1002/marc.200700529

18 M. Miya, R. Iwamoto, S. Yoshikawa and S. Mima, Int. J. Biol. Macromol., 2, 323 (1980), https://doi.org/10.1016/0141-8130(80)90056-2

19 B. Karimi and H. Seradj, Synlett, 4, 519 (2001)

${ }^{20}$ C. F. Liu, A. P. Zhang, W. Y. Li, F. X. Yue and R. C. Sun, J. Agric. Food Chem., 57, 1814 (2009), https://doi.org/10.1021/jf803097k

21 X. F. Sun, R. C. Sun and J. X. Sun, Bioresour. Technol., $\quad 95, \quad 343$ https://doi.org/10.1016/j.biortech.2004.02.025

22 X. F. Sun, R. C. Sun, J. Tomkinson and M. S. Baird, Carbohyd. Polym., 53, 483 (2003), https://doi.org/10.1016/S0144-8617(03)00150-4 